\title{
Posturographic pattern of patients with chronic subjective dizziness before and after vestibular rehabilitation
}

\author{
Benoît Morisod, Maxime Mermod and Raphaël Maire* \\ Department of Otorhinolaryngology, Head and Neck Surgery, Neurotology Unit, Lausanne \\ University Hospital, Switzerland
}

Received 3 June 2016

Accepted 19 October 2017

\begin{abstract}
.
INTRODUCTION: Chronic subjective dizziness (CSD) is frequently encountered in neurotology clinics. This diagnosis is mainly clinical, but computerized dynamic posturography (CDP) could be a helpful instrumental tool in the identification of these patients and validation of the treatment. This study was aimed to look for a specific posturographic pattern among patients diagnosed with CSD, and to eventually visualize improvement after vestibular rehabilitation.

METHODS: Single center, retrospective review from 2009 to 2014. We included patients diagnosed with CSD who underwent CDP in their neurotologic assessment. For those patients who benefited from vestibular rehabilitation, we compared their pre- and post-rehabilitation posturographies.

RESULTS: We included 114 patients, of whom $74 \%$ had known anxiety disorders and $33 \%$ a history of past vestibular disorder. $62 \%$ of the assessment posturographies were abnormal. The most affected sub-items were limit of stability, composite score of sensory organization tests and condition 5 in respectively $34 \%, 23 \%$ and $20 \%$ of the cases. In univariate analysis, only pathologic videonystagmography and history of unilateral vestibular dysfunction were significantly related to abnormal posturography. In the 42 patients who had vestibular rehabilitation and a post rehabilitation posturography, the proportion of abnormal posturography significantly dropped from $79 \%$ to $33 \%(p<0.001)$. When it was assessed, $79 \%$ of the patients reported a subjective improvement.
\end{abstract}

CONCLUSION: Patients with CSD have a high rate of abnormal posturography, but without a specific pattern. Vestibular rehabilitation is an effective tool in the therapeutic armamentarium.

Keywords: Chronic subjective dizziness, posturography, vestibular rehabilitation

\section{Introduction}

Chronic dizziness without any specific finding in standard neurotologic testing is a common situation [18]. It was often labeled as psychogenic, psychosomatic or even psychiatric dizziness. The strong relationship between anxiety disorders and vestibular disorders has now been well demonstrated

\footnotetext{
*Corresponding author: Dr. Raphaël Maire, Service d'ORL et de Chirurgie Cervico-Faciale, CHUV, Av. Du Bugnon 46, 1011 Lausanne, Switzerland. Tel.: +41213142716; Fax: +41213142646; E-mail: raphael.maire@chuv.ch.
}

$[2,13,24]$. As the understanding of the complex overlap between neurotology and psychiatry has evolved, these terms have been replaced by more specific diagnoses, like phobic postural vertigo [6] or chronic subjective dizziness (CSD) [9, 20]. Exacerbation of the symptoms in the presence of rich optokinetics stimuli (moving crowds or floors, supermarkets, action movies, specific or repetitive visual tasks like computer work) is frequently observed in this group of patients, and referred to as space and motion discomfort (SMD) [12] or visual vertigo [7]. This condition can potentially lead to avoidance 
behaviors and secondary phobias of the triggering conditions [31]. Most of these patients relate a history of past neurotologic disorder [26], or have either preexisting or quiescent anxiety disorders that have been triggered by the neurotologic injury [20]. Motion sickness and/or fear of heights are also frequently found in association [8]. An hypothesis is that in the central process of adaptation after a vestibular dysfunction, more weight is given to the "non vestibular" inputs, leading to visual or somatosensory dependence $[9,19,30]$. In the clinical assessment of patients with CSD, a posturography is often performed in addition to the standard otoneurologic tests. Our hypothesis is that patients with CSD could show a specific pattern on posturography, which may reflect a visual or somatosensory dependence. Treatment can include vestibular rehabilitation [15, 17], pharmacotherapy $[25,27]$ or psychotherapy $[3,11]$, depending on the presence or absence of vestibular lesion, severity of the psychiatric component, and patient's preference. As posturography has shown to be a potentially helpful tool in evaluating the effect of a treatment on postural control [29], we also compared the posturographic results before and after vestibular rehabilitation in the same population.

\section{Subjects and methods}

\subsection{Method}

We retrospectively reviewed the files of our neurotologic tertiary outpatient clinic from November 2009 to August 2014, and included all patients who underwent a computerized dynamic posturography (CDP) for CSD. We used the following diagnostic criteria for CSD as proposed by Staab in 2012 [22]: 1) chronic (>3 months) dizziness and/or unsteadiness that is present throughout the day but fluctuate in severity; 2) symptoms are related to body posture (most severe when moving, minor when recumbent); 3) exacerbation of the symptoms during motion, precision visual activities or in presence of rich optokinetics stimuli; 4) A triggering condition (acute or recurrent neurotologic disorder, medical condition or psychiatric disease that produce dizziness) is frequently encountered; 5) examination and vestibular testing may reveal diagnostic evidence of a neuro-otologic or other medical condition that may be active, treated, or resolved but cannot fully explain all of the patient's symptoms; 6) high prevalence of psychiatric disorders as anxiety or depression.

\subsection{Clinical assessment}

All CDP's were performed with the SmartEquitest $^{\circledR}$ (NeuroCom ${ }^{\circledR}$, USA). The results were recorded as normal or abnormal, according to the normative values provided by the system. All the results were interpreted by a single experienced neurotologist. The limit of stability (LOS) was considered pathologic if the patient failed in at least 2 quadrants. We looked for different subtypes of CSD, primarily vestibular (where Condition 5 and 6 are primarily affected) and non-vestibular deficits (where Conditions 1 and 2 are markedly below normal or Conditions 5 and 6 relatively better than Conditions 1 and 2, corresponding to criteria $\mathrm{n}^{\circ} 2$ and 3 of Mallinson and Longridge [16]). We also looked for aphysiologic sway pattern, using Criteria 5 of Mallinson and Longridge (circular sway (i.e. lateral and antero/posterior together) without any falls, and for a reduction of the stability limits around their centre of gravity and/or a displacement of the latter, suggestive of a sensory disorganization of the balance system [18].

All patients underwent a standard vestibular testing alongside the CDP (medical history and status, +/- bedside caloric testing, videonystagmography (VNG) including bithermal binaural caloric testing and video head impulse test recording, subjective visual vertical where the patient is asked to put a laser line vertical in a dark room, and a brain MRI). Canal paresis on caloric testing was diagnosed if an asymmetry greater than $20 \%$ was assessed. We recorded the age, sex, former vestibular rehabilitation, history of past vestibular disorder (e.g. benign paroxysmal positional vertigo (BPPV), unilateral peripheral vestibular deficit, Menière's disease), sudden sensorineural hearing loss (SSNHL), tinnitus, head concussion, previously diagnosed CSD, and history of past or current psychiatric disorders (anxiety, depression).

\subsection{Vestibular rehabilitation}

Vestibular rehabilitation (VR) was proposed as the first line of treatment. It was given by specialized physiotherapists, and consisted of a least one session per week, with daily exercises to practice at home. General standard equilibrium and re-afferentation exercises were performed, but the accent was made on individualization of the therapy, with in-situation exercises and cognitive-behavior therapy if needed. To assess the evolution, a CDP was proposed by 
the therapist at the end of the treatment regardless of the results. We retrospectively looked at the posturographic results before and after vestibular rehabilitation in the sub-group of patients that had both. The physiotherapist also most often recorded the subjective outcome self-reported by the patient, but no specific scale was used due to the retrospective design of the study.

\subsection{Statistical analysis}

Statistical analysis was performed using $\mathrm{R}$ software ( $\mathrm{R}$ Foundation for Statistical Computing, Vienna, Austria). Univariate analysis was performed using analysis of variance for continuous variables and fisher exact tests for categorical variables. Tests were 2 -sided, with significance set at $p<0.05$.

\section{Results}

One hundred and fourteen patients were included. There were 75 female and 39 male. Mean age was 47 years (range 27-75). Eighty-four patients were previously diagnosed with anxiety disorder (74\%) and $38(33 \%)$ for past vestibular or cochlear disorder (29 patients with unilateral peripheral vestibular deficit, 6 with BPPV and 3 with idiopathic SSNHL). Twelve patients were previously diagnosed with CSD $(11 \%)$. Thirteen patients had a history of minor cerebral concussion (11\%), and 8 an annoying tinnitus (7\%). Eighteen patients (16\%) already had former vestibular rehabilitation.

One hundred and twelve patients (98\%) had a bedside caloric test which was pathologic in 9 cases (8\%), and 107 (94\%) underwent a VNG, which was pathologic in 16 cases $(15 \%)$. It revealed a unilateral canal paresis in 14 cases. In two patients, a significant deviation of subjective visual vertical $\left(>2,5^{\circ}\right)$ was observed. Ninety-three (82\%) underwent a brain MRI, none of whom were abnormal. Patients' characteristics and association with abnormal CDP's are summarized in Table 1.

\subsection{Assessment posturography}

Seventy-one out of 114 (62\%) of the assessment CDP's were abnormal (i.e, the patient failed at least in one condition of the test). Distribution of the abnormal findings is summarized in Fig. 1. The most frequent pathologic items were the limit of stability (LOS) in $34 \%$, the composite score of the sensory organization test (SOT) in 23\%, and condition 5 (20\%) and 4 (19\%). In univariate analysis, only patients with a pathologic VNG $(p=0.024)$ and a history of unilateral vestibular dysfunction $(p<0.0001$, Fisher's tests) were more likely to have an abnormal posturography. Looking at different subtypes, we identified 9 patients (8\%) with Conditions 1 and 2 markedly below normal, and 8 patients $(7 \%)$ with Conditions 5 and 6 relatively better than Conditions 1 and 2. Twenty-six (23\%) patients showed a typical vestibular pattern where Condition 5 and 6 are primarily affected. We found 80 patients out of the $114(70 \%)$ with a shrinkage and/or displacement of their centre of gravity. 40 of them had solely a displacement of the center of gravity (35\%), 19 had a shrinkage only (17\%), and 21 (18\%) had both. The center of gravity was displaced posteriorly in 28 and anteriorly in 27, with only 6 displaced lateraly. None of these subtypes were significantly related to any of the clinical or instrumental variables.

\subsection{Vestibular rehabilitation and post rehabilitation posturography}

Eighty-five patients (75\%) underwent VR, with a mean of 10 sessions per patient (range 1-27). Fortytwo of those $(37 \%)$ had a post rehabilitation CDP, with a mean interval of 5,4 months between the two CDPs (range, 2-25 months). In the group of patients that had both CDP's, 14 (33\%) of the post rehabilitation posturographies were abnormal, versus $33(79 \%)$ initially (Chi2 $(1, \mathrm{~N}=43)=15.0465$, $p<0.0001)$. Twenty-four of that 42 patients $(57 \%)$ showed an improvement or normalization of their CDP, 13 (31\%) no changes and 5 (12\%) a worsening (Fig. 2). The most frequent abnormal items on the post rehabilitation CDP were composite score and strategy analysis (21\%), and LOS and condition $6(17 \%)$. Statistical analysis revealed only a significant reduction of abnormal LOS (48\% vs $17 \%, p=0.0046)$ and condition $5(38 \%$ vs $12 \%$, $p=0.0107$, Fisher's tests) between the two CDP's (Fig. 3). Abnormal post rehabilitation CDP's were not statistically associated with any of the demographic or clinical parameters (Table 1). Subgroup analysis showed that patients with a head concussion is the only patient's subset with a significant amelioration of the post VR CDP $(p=0.0257$, Fisher's test).

On the 85 patients that had VR, the subjective outcome was assessed by the physical therapist in 53 
Table 1

Patients' demographics and their association with abnormal posturographies

\begin{tabular}{|c|c|c|c|}
\hline Patients & $\mathrm{N}=114(\%)$ & $\begin{array}{l}\text { Association with } \\
\text { abnormal } \\
\text { assessment CDP }\end{array}$ & $\begin{array}{c}\text { Association with } \\
\text { abnormal } \\
\text { post-VR CDP }\end{array}$ \\
\hline \multicolumn{4}{|l|}{ Demographics } \\
\hline Median age & 47 years (range $27-75$ ) & NS & NS \\
\hline Female/Male & $75 / 39(66 / 34)$ & NS & NS \\
\hline Anxiety disorders & $84(74)$ & NS & NS \\
\hline Previously diagnosed CSD & $12(11)$ & NS & NS \\
\hline Previous vestibular rehabilitation & $18(16)$ & NS & NS \\
\hline Minor head concussion & $13(11)$ & NS & NS \\
\hline Annoying tinnitus & $8(7)$ & NS & NS \\
\hline History of neuro-otologic dysfunction & $38(33)$ & & \\
\hline Unilateral vestibular dysfunction & $29(25)$ & $p$-value $<0.0001$ & NS \\
\hline BPPV & $6(5)$ & NS & NS \\
\hline SSNHL & $3(3)$ & NS & NS \\
\hline \multicolumn{4}{|l|}{ Vestibular testing } \\
\hline Bedside caloric tests (abnormal/done) & $9 / 112(8)$ & NS & NS \\
\hline VNG (including BCT/VHIT) (abnormal/done) & $16 / 107(15)$ & $p$-value $=0.0244$ & NS \\
\hline MRI (abnormal/done) & $0 / 93(0)$ & NA & NA \\
\hline
\end{tabular}

CSD: chronic subjective dizziness; CDP: computerized dynamic posturography; VR: vestibular rehabilitation; BPPV: benign positional paroxystic vertigo; SSNHL: sudden sensorineural hearing loss; VNG: videonystagmography; BCT : bithermal caloric testing; VHIT : video head impulse test; MRI: magnetic resonance imaging. NS: not statistically significant. NA: not applicable.

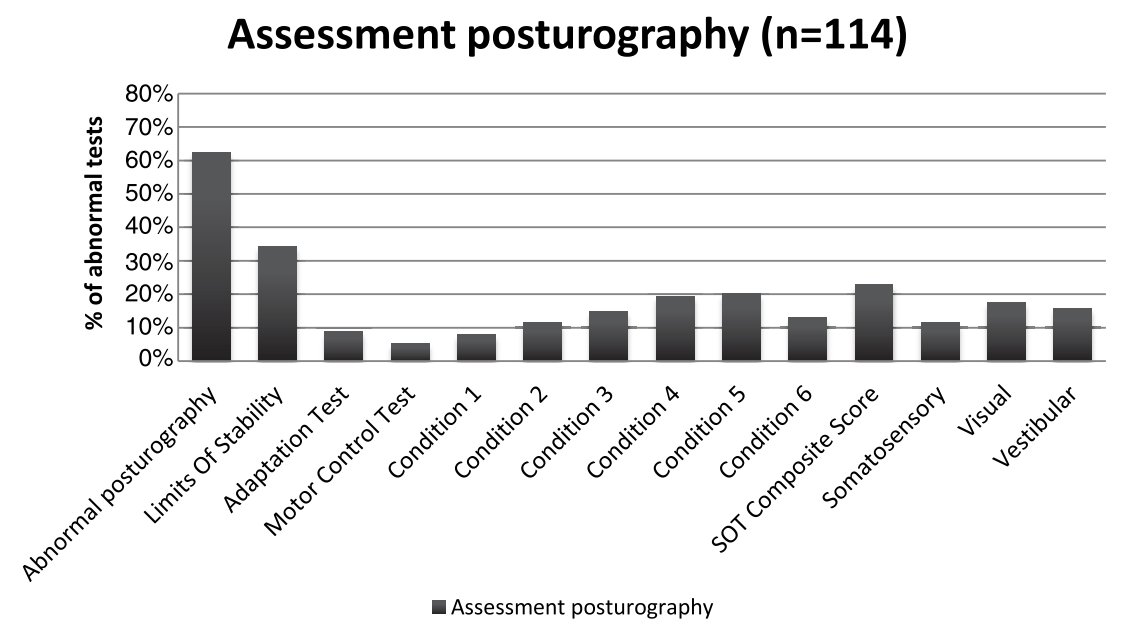

Fig. 1. Distribution of abnormal tests on assessment posturography.

cases (62\%). Forty-two patients (79\%) self-reported an improvement and $11(21 \%)$ no changes or a worsening (Fig. 2).

\section{Discussion}

Our sample of patients presenting with CSD shows a female preponderance and a high rate of concomitant anxiety disorders. A third of them also had a past history of vestibular lesion, and $15 \%$ still show a unilateral vestibular deficit on VNG testing. All of this is in line with existing literature on CSD [13, $20,22,24]$. We hypothesized that a high rate of abnormal CDP would be observed, reflecting a visual or somatosensory dependence. In previous studies, Jacob et al. [14] have found a statistically significant correlation between space and motion discomfort and somatosensory dependence (condition 4), but inconclusive data for visual dependence (condition 3). Tjernström et al. [28] showed that patients with phobic postural vertigo adapt to proprioceptive perturbation to a lesser extent than normal subjects, and that they do not use visual information as efficiently 


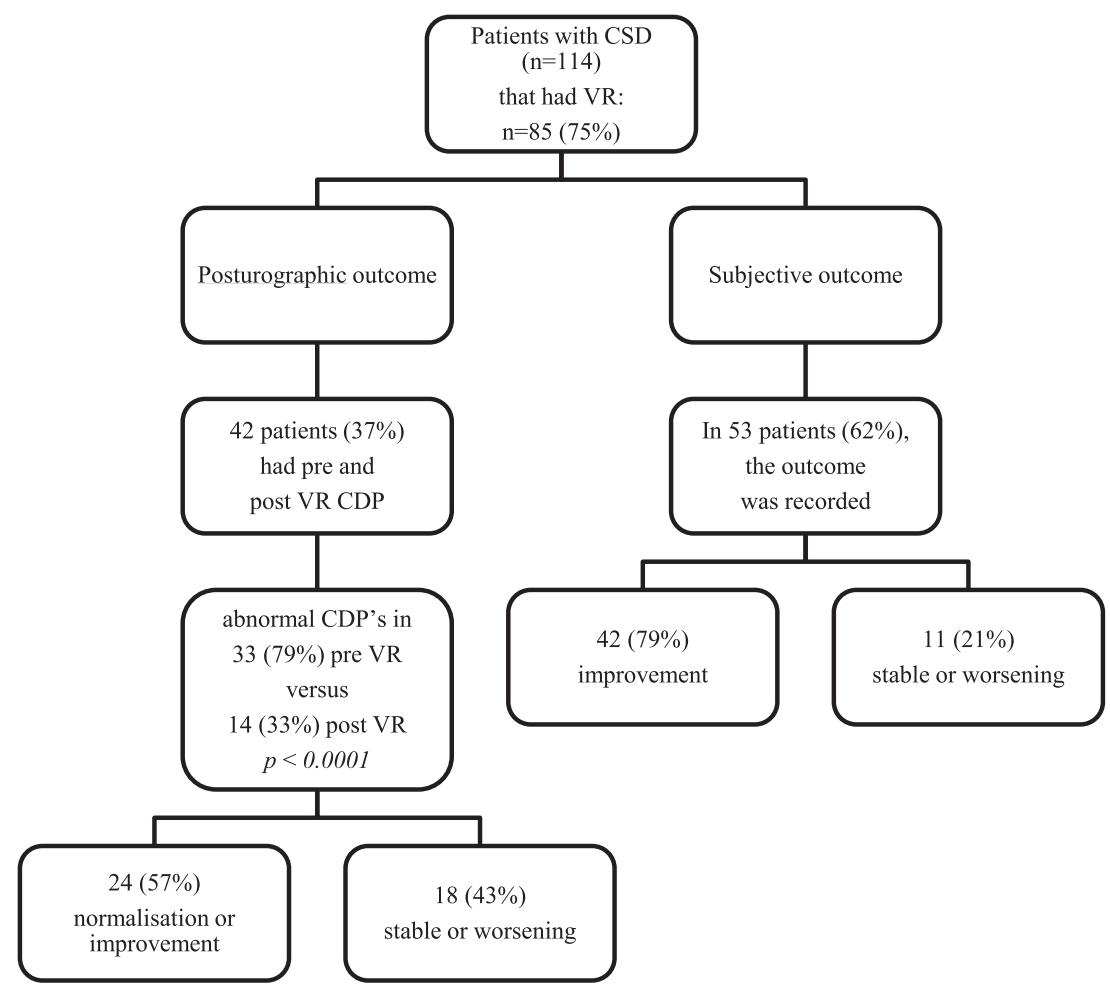

Fig. 2. Posturographic and subjective outcome of patients with chronic subjective dizziness that had vestibular rehabilitation. CSD: chronic subjective dizziness; VR: vestibular rehabilitation; CDP: computerized dynamic posturography.

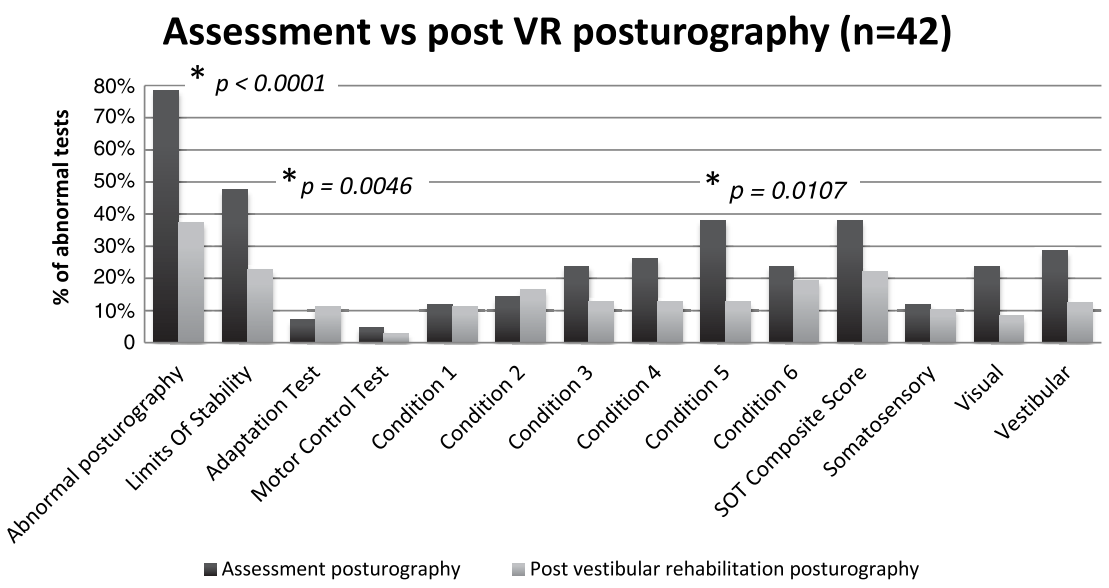

Fig. 3. Distribution of abnormal tests on assessment and post-vestibular rehabilitation posturographies: Significant improvement was found on Chi2 and Fischer test for the rate of abnormal posturography, LOS and condition 5 (*: statistically significant improvement, p-value).

to modulate postural control. Our study confirms that patients with CSD present with an abnormal balance on the CDP, but failed to find a pattern specific to that condition. Abnormal posturographies (62\%) were only significantly associated with a unilateral peripheral vestibular deficit, either on VNG or at history (Table 1). Nevertheless, their patterns differ from the typical vestibular pattern in which condition 5 and 6 are primarily affected. In our study, the most frequently affected items were LOS, (34\%), the composite score (23\%) and condition $5(20 \%)$ and $4(19 \%)$. As seen in Fig. 1, there are no signifi- 
cant differences between the various posturographic conditions. These findings are more suggestive of an aspecific pattern.

We also looked for various sub-types, primarily vestibular versus non-vestibular patterns, using criteria 2, 4 and 5 of Mallinson and Longridge. Some of the patients distinctly showed either a vestibular (where C5 and C6 are primarily affected) or a non vestibular pattern $(\mathrm{C} 1$ and $\mathrm{C} 2$ markedly lower than normal or $\mathrm{C} 5$ and $\mathrm{C} 6$ relatively better than $\mathrm{C} 1$ and $\mathrm{C} 2$ ), but that was not linked in a significant matter to any of the clinical or instrumental assessments, notably to vestibular deficits. We found a few patients with a positive $\mathrm{n}^{\circ} 5$ criteria of Mallinson and Longridge, but none of these subtypes where in sufficient number to conclude that they are indicative of CSD.

$80 \%$ of our patients showed a shrinkage and/or a displacement of their centre of gravit, numbers that are similar to previous published data [18]. Together with the high amount of abnormal LOS we found, it suggests a sensory disorganization of the balance system with inappropriate responses in postural control. Another hypothesis could be a fear to fall during the testing. Indeed our patient sample has a high rate of concomitant anxiety disorders. A correlation between anxiety disorders and abnormal posturographic findings, mostly enhanced antero-posterior sway, has already been reported [10]. Redfern et al. [19] found a greater sway in response to moving visual environment in anxious patients with space and motion disorder (SMD) in comparison to healthy subjects, but also in comparison to anxious patients without SMD. They conclude that patients with anxiety disorders are more visually dependent for balance. In our study, patients with known or treated anxiety disorders didn't performed worse than non anxious patients.

A recent study compared SOT scores of patients with persistent postural-perceptual dizziness (PPPD) with normal control subjects and recovered vestibular patients [21]. PPPD was recently described by the Barany Society and its diagnostic criteria are derived from phobic postural vertigo and CSD [23]. Our data was recorded before this entity was described, but PPPD should be used as the generic term in future publications. This study showed that patients with PPPD perform poorer than subjects in the recovered group and control group, with greater deficits in mean scores across all SOT conditions except $\mathrm{C} 1$. They also came to the conclusion that poorer performances on SOT in these patients are probably caused by the confluence of three factors: excessive visual or somatosensory dependence, anxiety and use of high-risk postural strategies when not needed.

Our study is in line with these results; the high rate of abnormal posturographies and variability of results with many different subtypes among patients with CSD suggest a sensory disorganization of their balance system that finally leads to various responses in term of postural control. We think that all the parameters that could influence the posturographic results (prior vestibular disorder, visual or somatosensory dependence, anxiety, use of high-risk postural strategies when they are not needed, ... ) can lead to various posturographic patterns, thus failing to identify a specific pattern for this condition.

Vestibular therapy has already shown its value in the treatment of vestibular disorders [4, 5]. In our study, the post VR CDP's showed a significant improvement compared to the assessment ones (79\% abnormal initially vs $33 \%$ post rehabilitation, Fig. 2). It confirms the value of this type of treatment for CSD. Patients with head concussion syndrome seem to benefit the most. In a randomized control trial, Andersson et al. [1] found a significant improvement in the self-reported dizziness handicap inventory and the vertigo symptom scale in patients that had VR plus cognitive-behavior therapy compared to controls, which showed no improvement at all. Due to its retrospective design, our study lacks a control group, so the improvement rate is to take with caution. Nevertheless, $57 \%$ of the patients that had a VR demonstrated an improvement on the CDP, a response rate that is comparable to sertraline treatment in another study [27]. Most importantly, 79\% of the patients reported a subjective improvement when this parameter has been assessed (Fig. 2). The post-VR posturography also allows the physical therapist and the patient to objective and validate the progresses, or to accept failure of the treatment and the need for a complementary approach. Thus we recommend VR as the first line treatment for CSD, considering its reasonable efficacy without potential secondary effects as compared to pharmacological treatment. Moreover, it can include do-it-at-home, insituation and cognitive-behavioral exercises, offering a wide room for individualization of the treatment. Nevertheless, a psychiatric evaluation in addition to vestibular testing is advocated in cases of major psychiatric disorders and phobia, where combined treatments and multidisciplinary approach is mandatory. 


\section{Conclusion}

Patients with chronic subjective dizziness have a high rate of abnormal balance test, without a specific pattern on the computerized dynamic posturography. Vestibular rehabilitation is an effective tool in the therapeutic armamentarium.

\section{Conflicts of interest}

The authors declare that there are no conflicts of interest.

\section{References}

[1] G. Andersson, G.J. Asmundson, J. Denev, J. Nilsson and H.C. Larsen, A controlled trial of cognitive-behavior therapy combined with vestibular rehabilitation in the treatment of dizziness, Behav Res Ther 44 (2006), 1265-1273.

[2] G.J. Asmundson, D.K. Larsen and M.B. Stein, Panic disorder and vestibular disturbance: An overview of empirical findings and clinical implications, J Psychosom Res 44 (1998), 107-120.

[3] D.C. Beidel and F.B. Horak, Behavior therapy for vestibular rehabilitation, J Anxiety Disord 15 (2001), 121-130.

[4] R.S. Bittar, M.E. Pedalini, J.O. Ramalho and R. Yoshimura, Critical analysis of vestibular rehabilitation outcome according to dizziness etiology, Braz J Otorhinolaryngol 73 (2007), 760-764.

[5] F.C. Boyer, L. Percebois-Macadre, E. Regrain, M. Leveque, R. Taiar, L. Seidermann, G. Belassian and A. Chays, Vestibular rehabilitation therapy, Neurophysiol Clin $\mathbf{3 8}$ (2008), 479-487.

[6] T. Brandt, Phobic postural vertigo, Neurology 46 (1996), 1515-1519.

[7] A.M. Bronstein, The visual vertigo syndrome, Acta Otolaryngol Suppl 520(Pt 1) (1995), 45-48.

[8] D.B. Clark, B.E. Hirsch, M.G. Smith, J.M. Furman and R.G. Jacob, Panic in otolaryngology patients presenting with dizziness or hearing loss, Am J Psychiatry 151 (1994), 1223-1225.

[9] J.M. Furman and R.G. Jacob, A clinical taxonomy of dizziness and anxiety in the otoneurological setting, $J$ Anxiety Disord 15 (2001), 9-26.

[10] F. Goto, M. Kabeya, K. Kushiro, T. Ttsutsumi and K. Hayashi, Effect of anxiety on antero-posterior postural stability in patients with dizziness, Neurosci Lett 487 (2011), 204-206.

[11] J. Holmberg, M. Karlberg, U. Harlacher, M. Rivano-Fischer and M. Magnusson, Treatment of phobic postural vertigo. A controlled study of cognitive-behavioral therapy and self-controlled desensitization, J Neurol 253 (2006), 500-506.

[12] R. Jacob, S. Woody, D. Clark, S. Lilienfeld, B. Hirsch, G. Kucera, J. Furman and J. Durrant, Discomfort with space and motion: A possible marker of vestibular dysfunction assessed by the Situational Characteristics Questionnaire,
Journal of Psychopathology and Behavioral Assessment 15 (1993), 299-324.

[13] R.G. Jacob and J.M. Furman, Psychiatric consequences of vestibular dysfunction, Curr Opin Neurol 14 (2001), 41-46.

[14] R.G. Jacob, M.S. Redfern and J.M. Furman, Space and motion discomfort and abnormal balance control in patients with anxiety disorders, J Neurol Neurosurg Psychiatry $\mathbf{8 0}$ (2009), 74-78.

[15] R.G. Jacob, S.L. Whitney, G. Detweiler-Shostak and J.M. Furman, Vestibular rehabilitation for patients with agoraphobia and vestibular dysfunction: A pilot study, J Anxiety Disord 15 (2001), 131-146.

[16] A.I. Mallinson and N.S. Longridge, A new set of criteria for evaluating malingering in work-related vestibular injury, Otol Neurotol 26 (2005), 686-690.

[17] A. Meli, G. Zimatore, C. Badaracco, E. De Angelis and D. Tufarelli, Effects of vestibular rehabilitation therapy on emotional aspects in chronic vestibular patients, $J$ Psychosom Res 63 (2007), 185-190.

[18] M. Odman and R. Maire, Chronic subjective dizziness, Acta Otolaryngol 128 (2008), 1085-1088.

[19] M.S. Redfern, J.M. Furman and R.G. Jacob, Visually induced postural sway in anxiety disorders, J Anxiety Disord 21 (2007), 704-716.

[20] M.J. Ruckenstein and J.P. Staab, Chronic subjective dizziness, Otolaryngol Clin North Am 42 (2009), 71-77, ix.

[21] E. Sohsten, R.S. Bittar and J.P. Staab, Posturographic profile of patients with persistent postural-perceptual dizziness on the sensory organization test, J Vestib Res 26 (2016), 319-326.

[22] J.P. Staab, Chronic subjective dizziness, Continuum (Minneap Minn) 18 (2012), 1118-1141.

[23] E.-H.A. Staab, A. Horii, R. Jacob and M. Strupp, Progress report of the Behavioral Subcommittee of the Committee on Classification of the Bárány Society, J Vestib Res 24 (2014), 93-94.

[24] J.P. Staab and M.J. Ruckenstein, Which comes first? Psychogenic dizziness versus otogenic anxiety, Laryngoscope 113 (2003), 1714-1718.

[25] J.P. Staab and M.J. Ruckenstein, Chronic dizziness and anxiety: Effect of course of illness on treatment outcome, Arch Otolaryngol Head Neck Surg 131 (2005), 675-679.

[26] J.P. Staab and M.J. Ruckenstein, Expanding the differential diagnosis of chronic dizziness, Arch Otolaryngol Head Neck Surg 133 (2007), 170-176.

[27] J.P. Staab, M.J. Ruckenstein and J.D. Amsterdam, A prospective trial of sertraline for chronic subjective dizziness, Laryngoscope 114 (2004), 1637-1641.

[28] F. Tjernstrom, P.A. Fransson, J. Holmberg, M. Karlberg and M. Magnusson, Decreased postural adaptation in patients with phobic postural vertigo-an effect of an "anxious" control of posture? Neurosci Lett 454 (2009), 198-202.

[29] J.E. Visser, M.G. Carpenter, H. van der Kooij and B.R. Bloem, The clinical utility of posturography, Clin Neurophysiol 119 (2008), 2424-2436.

[30] S.L. Whitney, R.G. Jacob, P.J. Sparto, E.F. Olshansky, G. Detweiler-Shostak, E.L. Brown and J.M. Furman, Acrophobia and pathological height vertigo: Indications for vestibular physical therapy? Phys Ther $\mathbf{8 5}$ (2005), 443-458.

[31] L. Yardley, J. Britton, S. Lear, J. Bird and L.M. Luxon, Relationship between balance system function and agoraphobic avoidance, Behav Res Ther 33 (1995), 435-439. 